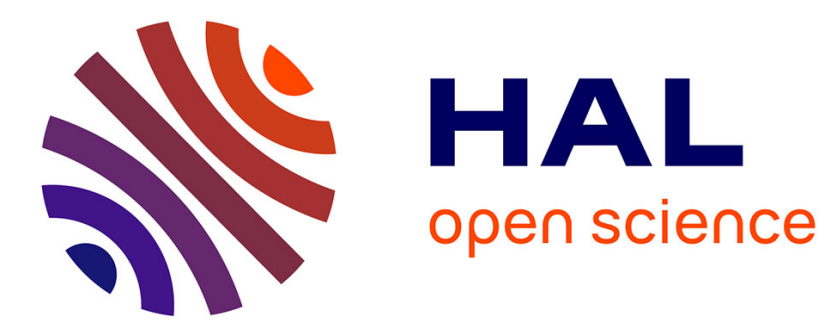

\title{
On conditions of robust synchronization for multistable systems
}

Hafiz Ahmed, Rosane Ushirobira, Denis Efimov, Wilfrid Perruquetti

\section{To cite this version:}

Hafiz Ahmed, Rosane Ushirobira, Denis Efimov, Wilfrid Perruquetti. On conditions of robust synchronization for multistable systems. Proc. ECC'15, Jul 2015, Linz, Austria. hal-01140326

\section{HAL Id: hal-01140326 \\ https://hal.inria.fr/hal-01140326}

Submitted on 8 Apr 2015

HAL is a multi-disciplinary open access archive for the deposit and dissemination of scientific research documents, whether they are published or not. The documents may come from teaching and research institutions in France or abroad, or from public or private research centers.
L'archive ouverte pluridisciplinaire HAL, est destinée au dépôt et à la diffusion de documents scientifiques de niveau recherche, publiés ou non, émanant des établissements d'enseignement et de recherche français ou étrangers, des laboratoires publics ou privés. 


\title{
On conditions of robust synchronization for multistable systems
}

\author{
Hafiz Ahmed, Rosane Ushirobira, Denis Efimov and Wilfrid Perruquetti
}

\begin{abstract}
This paper deals with a robust synchronization problem for multistable systems evolving on manifolds in the context of Input-to-State Stability (ISS) framework. Based on a recent generalization of classical ISS theory to multistable systems (may have unstable equilibrium), a robust synchronization protocol is designed with respect to a compact invariant set of the unperturbed system. The invariant set is assumed to admit a decomposition without cycles (basically no homoclinic nor heteroclinic orbits may exist). Numerical simulation examples illustrate our theoretical results.
\end{abstract}

\section{INTRODUCTION}

The first scientific observation regarding a synchronization event can be dated back to seventeenth century by a Dutch scientist C. Huygens, who observed a synchronous behavior in coupled pendulums [1]. This type of behavior can be seen in a variety of systems in various domains, for example biological, chemical, mechanical, etc. [2], [3], [4], [5]. Synchronization of complex dynamical systems and/or network of systems has attracted a lot of attention from multidisciplinary research communities over the last decades because of their pervasive presence in nature, technology and human society. A collective behavior arises from the interconnection of dynamical systems and it has various potential application domains. For example, transient stability in power network [6], cooperative multitasking and formation control [7]. The core of synchronization is the collective objective of agents in a network to reach a consensus about certain variables of interest.

The existing literature on the synchronization problem is very vast and covers many areas. In [8], the problem of formation control is investigated in swarms within the framework of output regulation in nonlinear systems. Detailed study regarding the control and synchronization of chaos can be found in [9]. The paper [10] extends optimal control and adaptive control design methods to multi-agent nonlinear systems on communication graphs. Recent advances in various aspects of cooperative control of multi-agent systems can be found in

The authors are with the Non-A team @ Inria, Parc Scientifique de la Haute Borne, 40 avenue Halley, 59650 Villeneuve d'Ascq, France. Denis Efimov and Wilfrid Perruquetti are with CRIStAL UMR 9189, Ecole Centrale de Lille, Avenue Paul Langevin, 59651 Villeneuve d'Ascq, France. E-mail: \{hafiz.ahmed, rosane.ushirobira, denis.efimov, wilfrid.perruquetti @ inria.fr\}

D. Efimov is with Department of Control Systems and Informatics, Saint Petersburg State University of Information Technologies Mechanics and Optics (ITMO), 49 av. Kronverkskiy, 197101 Saint Petersburg, Russia.

Hafiz Ahmed is partly supported by the regional council of Nord-Pas de Calais, France.

This work was supported in part by Région Nord-Pas de Calais, France, by the Government of Russian Federation (Grant 074-U01) and the Ministry of Education and Science of Russian Federation (Project 14.Z50.31.0031).
[11]. The theoretical framework for design and analysis of distributed flocking algorithms can be found in [12].

In this work, we consider the synchronization problem for multistable systems based on the framework of ISS. This is a very well established technique for the study of stability and robustness of nonlinear systems. The ISS property provides a natural framework of stability analysis with respect to input perturbations (see [13] and references therein). The classical definition allows stability properties with respect to arbitrary compact invariant sets (and not simply equilibria) to be formulated and characterized. However, the implicit requirement that these sets should be simultaneously Lyapunov stable and globally attractive, makes the basic theory not applicable for a global analysis of many dynamical behaviors of interest, having multistability [14], [15], [16], periodic oscillations [17] or hidden attractors [18], just to name a few, and only local analysis remains possible [19]. Attempts were made to overcome such limitations by introducing the notions of almost global stability [20] and almost input-to-state stability [21], etc.

Recently, the authors in [22] have proposed that the most natural way of relaxing ISS condition for systems with multiple invariant sets is equivalent to relax the Lyapunov stability requirement [23] (rather than the global nature of the attractivity property). Using this relatively mild condition, the authors [22] have generalized the ISS theory as well as, the related literature on time invariant autonomous dynamical systems on compact spaces [24] for multistable systems. In our current work, the results presented in [22] are extended to provide sufficient conditions for the existence of robust synchronization for multistable systems.

The rest of the paper is organized as follows. Section II introduces some preliminaries about decomposable sets and the notions of robustness with respect to ISS. Our main results and the family of nonlinear systems being considered can be found in section III. Numerical simulation examples are given to illustrate these results in section IV. The paper closes with some concluding remarks in section $\mathrm{V}$.

\section{PREliminaries}

For an $n$-dimensional $\mathcal{C}^{2}$ connected and orientable Riemannian manifold $M$ without a boundary, let the map $f(x, d)$ : $M \times \mathbb{R}^{m} \rightarrow T_{x} M$ be of class $\mathcal{C}^{1}$, and consider a nonlinear system of the following form:

$$
\dot{x}(t)=f(x(t), d(t))
$$

where the state $x \in M$ and $d(t) \in \mathbb{R}^{m}$ (the input $d(\cdot)$ is a locally essentially bounded and measurable signal) for $t \geq 0$. 
We denote by $X(t, x ; d(\cdot))$ the uniquely defined solution of (1) at time $t$ fulfilling $X(0, x ; d(\cdot))=x$. Together with (1) we will analyze its unperturbed version:

$$
\dot{x}(t)=f(x(t), 0) .
$$

A set $S \subset M$ is invariant for the unperturbed system (2) if $X(t, x ; 0) \in S$ for all $t \in \mathbb{R}$ and for all $x \in S$. For a set $S \subset M$ define the distance to the set $|x|_{S}=\min _{a \in S} \delta(x, a)$ from a point $x \in M$, where the symbol $\delta\left(x_{1}, x_{2}\right)$ denotes the Riemannian distance between $x_{1}$ and $x_{2}$ in $M,|x|=|x|_{\{0\}}$ for $x \in M$ or the usual Euclidean norm of a vector $x \in \mathbb{R}^{n}$. For a signal $d: \mathbb{R} \rightarrow \mathbb{R}^{m}$ the essential supremum norm is defined as $\|d\|_{\infty}=\operatorname{ess~sup}_{t>0}|d(t)|$.

A function $\alpha: \mathbb{R}_{+} \rightarrow \mathbb{R}_{+}$is said to belong to class $\mathcal{K}$, i.e., $\alpha \in \mathcal{K}$, if it is continuous, strictly increasing and $\alpha(0)=0$. Furthermore, $\alpha \in \mathcal{K}_{\infty}$ if $\alpha \in \mathcal{K}$ and it is unbounded, i.e. $\lim _{s \rightarrow \infty} \alpha(s)=\infty$.

For any points $x, y \in M$, the $\alpha$ and $\omega$ - limit sets for (2) can be defined as follows:

$$
\begin{aligned}
& \alpha(x):=\left\{y \mid y=\lim _{n \rightarrow-\infty} X\left(x, t_{n}\right) \text { where } t_{n} \searrow-\infty\right\} \\
& \omega(x):=\left\{y \mid y=\lim _{n \rightarrow \infty} X\left(x, t_{n}\right) \text { where } t_{n} \nearrow \infty\right\} .
\end{aligned}
$$

\section{A. Decomposable sets}

Let $\Lambda \subset M$ be a compact invariant set for (2).

Definition 1. [24] A decomposition of $\Lambda$ is a finite and disjoint family of compact invariant sets $\Lambda_{1}, \ldots, \Lambda_{k}$ such that

$$
\Lambda=\bigcup_{i=1}^{k} \Lambda_{i} .
$$

For an invariant set $\Lambda$, its attracting and repulsing subsets are defined as follows:

$$
\begin{aligned}
W^{s}(\Lambda) & =\left\{x \in M:|X(t, x, 0)|_{\Lambda} \rightarrow 0 \text { as } t \rightarrow+\infty\right\}, \\
W^{u}(\Lambda) & =\left\{x \in M:|X(t, x, 0)|_{\Lambda} \rightarrow 0 \text { as } t \rightarrow-\infty\right\} .
\end{aligned}
$$

Define a relation on the family of invariants sets of $M$ : for $\mathcal{W} \subset M$ and $\mathcal{D} \subset M$, we write $\mathcal{W} \prec \mathcal{D}$ if $W^{s}(\mathcal{W}) \cap W^{u}(\mathcal{D}) \neq \oslash$.

Definition 2. [24] Let $\Lambda_{1}, \ldots, \Lambda_{k}$ be a decomposition of $\Lambda$, then

1. An $r$-cycle $(r \geq 2)$ is an ordered $r$-tuple of distinct indices $i_{1}, \ldots, i_{r}$ such that $\Lambda_{i_{1}} \prec \ldots \prec \Lambda_{i_{r}} \prec \Lambda_{i_{1}}$.

2. A 1 -cycle is an index $i$ such that $\left[W^{u}\left(\Lambda_{i}\right) \cap W^{s}\left(\Lambda_{i}\right)\right]-$ $\Lambda_{i} \neq \varnothing$.

3. A filtration ordering is a numbering of the $\Lambda_{i}$ so that $\Lambda_{i} \prec \Lambda_{j} \Rightarrow i \leq j$.

As we can conclude from Definition 2, the existence of an $r$ cycle with $r \geq 2$ is equivalent to the existence of a heteroclinic cycle for (2) [25]. And the existence of a 1-cycle implies the existence of a homoclinic cycle for (2) [25].

Let a compact set $\mathcal{W} \subset M$ be containing all $\alpha$ and $\omega$ limit sets of (2).

Definition 3. The set $\mathcal{W}$ is called decomposable if it admits a finite decomposition without cycles, $\mathcal{W}=\bigcup_{i=1}^{k} \mathcal{W}_{i}$, for some non-empty disjoint compact sets $\mathcal{W}_{i}$, which form a filtration ordering of $\mathcal{W}$, as detailed in definitions 1 and 2 .

\section{B. Robustness notions}

The following robustness notions for systems in (1) have been introduced in [22].

Definition 4. We say that the system (1) has the practical asymptotic gain (pAG) property if there exist $\eta \in \mathcal{K}_{\infty}$ and a non-negative real $q$ such that for all $x \in M$ and all measurable essentially bounded inputs $d(\cdot)$ the solutions are defined for all $t \geq 0$ and the following holds:

$$
\limsup _{t \rightarrow+\infty}|X(t, x ; d)|_{\mathcal{W}} \leq \eta\left(\|d\|_{\infty}\right)+q .
$$

If $q=0$, then we say that the asymptotic gain (AG) property holds.

Definition 5. We say that the system (1) has the limit property (LIM) with respect to $\mathcal{W}$ if there exists $\mu \in \mathcal{K}_{\infty}$ such that for all $x \in M$ and all measurable essentially bounded inputs $d(\cdot)$ the solutions are defined for all $t \geq 0$ and the following holds:

$$
\inf _{t \geq 0}|X(t, x ; d)|_{\mathcal{W}} \leq \mu\left(\|d\|_{\infty}\right) .
$$

Definition 6. We say that the system (1) has the practical global stability (pGS) property with respect to $\mathcal{W}$ if there exist $\beta \in \mathcal{K}_{\infty}$ and $q \geq 0$ such that for all $x \in M$ and all measurable essentially bounded inputs $d(\cdot)$ the following holds for all $t \geq$ 0 :

$$
|X(t, x ; d)|_{\mathcal{W}} \leq q+\beta\left(\max \left\{|x|_{\mathcal{W}},\|d\|_{\infty}\right\}\right) .
$$

It has been shown in [22] that to characterize (3) in terms of Lyapunov functions the following notion is appropriate:

Definition 7. We say that a $\mathcal{C}^{1}$ function $V: M \rightarrow \mathbb{R}$ is a practical ISS-Lyapunov function for (1) if there exists $\mathcal{K}_{\infty}$ functions $\alpha_{1}, \alpha_{2}, \alpha$ and $\gamma$, and scalars $q \geq 0$ and $c \geq 0$ such that

$$
\alpha_{1}\left(|x|_{\mathcal{W}}\right) \leq V(x) \leq \alpha_{2}\left(|x|_{\mathcal{W}}+c\right),
$$

the function $V$ is constant on each $\mathcal{W}_{i}$ and the dissipation inequality below holds:

$$
D V(x) f(x, d) \leq-\alpha\left(|x|_{\mathcal{W}}\right)+\gamma(|d|)+q .
$$

If the dissipation inequality holds for $q=0$, then $V$ is said to be an ISS-Lyapunov function.

Notice that existence of $\alpha_{2}$ and $c$ follows (without any additional assumptions) by standard continuity arguments.

The main result of [22] connecting these robust stability properties is states below:

Theorem 8. Consider a nonlinear system as in (1) and let a compact invariant set containing all $\alpha$ and $\omega$ limit sets of (2) $\mathcal{W}$ be decomposable (in the sense of Definition 3). Then the following facts are equivalent.

1. The system admits an ISS Lyapunov function.

2. The system enjoys the AG property.

3. The system admits a practical ISS Lyapunov function.

4. The system enjoys the $p A G$ property.

5. The system enjoys the LIM property and the $p G S$.

A system (1) for which this list of equivalent properties is satisfied, is called ISS with respect to the set $\mathcal{W}$ [22]. 


\section{SYNCHRONIZATION OF MULTISTABLE SYSTEMS}

In this section we will consider the following family of nonlinear systems:

$$
\dot{x}_{i}(t)=f_{i}\left(x_{i}(t), u_{i}(t), d_{i}(t)\right), i=1, \ldots, N, N>1,
$$

where the state $x_{i} \in M_{i}$ and $M_{i}$ is an $n_{i}$-dimensional $\mathcal{C}^{2}$ connected and orientable Riemannian manifold without a boundary, the control $u_{i}(t) \in \mathbb{R}^{m_{i}}$ and the external disturbance $d_{i}(t) \in \mathbb{R}^{p_{i}}\left(u_{i}(\cdot)\right.$ and $d_{i}(\cdot)$ are locally essentially bounded and measurable signals) for $t \geq 0$, the map $f_{i}\left(x_{i}, u_{i}, d_{i}\right)$ : $M_{i} \times \mathbb{R}^{m_{i}} \times \mathbb{R}^{p_{i}} \rightarrow T_{x} M_{i}$ is $\mathcal{C}^{1}, f_{i}(0,0,0)=0$. Denote the common state vector of (4) as $x=\left[x_{1}^{T}, \ldots, x_{N}^{T}\right]^{T} \in$ $M=\prod_{i=1}^{N} M_{i}$ ( $M$ is the corresponding Riemannian manifold of dimension $n=\sum_{i=1}^{N} n_{i}$ where the family (4) behaves), $d=\left[d_{1}^{T}, \ldots, d_{N}^{T}\right]^{T} \in \mathbb{R}^{p}$ with $p=\sum_{i=1}^{N} p_{i}$ is the total exogenous input.

Assumption 1. For all $i=1, \ldots, N$, each system in (4) has a compact invariant set $\mathcal{W}_{i}$ containing all $\alpha$ and $\omega$ limit sets of $\dot{x}_{i}(t)=f_{i}\left(x_{i}(t), 0,0\right), \mathcal{W}_{i}$ is decomposable in the sense of Definition 3, and the system enjoys the AG property with respect to inputs $u_{i}$ and $d_{i}$ as in Definition 4.

In this case, from Theorem 8 , there exist $\mathcal{C}^{1}$ ISS-Lyapunov functions $V_{i}: M_{i} \rightarrow \mathbb{R}$ with $\mathcal{K}_{\infty}$ functions $\alpha_{1 i}, \alpha_{2 i}, \alpha_{3 i}, \gamma_{u i}$ and $\gamma_{d i}$ such that

$$
\begin{gathered}
\alpha_{1 i}\left(\left|x_{i}\right| \mathcal{W}_{i}\right) \leq V_{i}\left(x_{i}\right) \leq \alpha_{2 i}\left(\left|x_{i}\right| \mathcal{W}_{i}+c_{i}\right), c_{i} \geq 0, \\
D V_{i}\left(x_{i}\right) f_{i}\left(x_{i}, u_{i}, d_{i}\right) \leq-\alpha_{3 i}\left(\left|x_{i}\right| \mathcal{W}_{i}\right)+\gamma_{u i}\left(\left|u_{i}\right|\right)+\gamma_{d i}\left(\left|d_{i}\right|\right)
\end{gathered}
$$

for all $i=1, \ldots, N$. For a future reference, define also the invariant set of disconnected and unperturbed $\left(u_{i}=d_{i}=0\right)$ family $\mathcal{W}=\prod_{i=1}^{N} \mathcal{W}_{i} \subset M(0 \in \mathcal{W})$. Then, by definition, there exist functions $\nu_{1}, \nu_{2} \in \mathcal{K}_{\infty}$ such that

$$
\nu_{1}\left(|x|_{\mathcal{W}}\right) \leq \sum_{i=1}^{N}\left|x_{i}\right|_{\mathcal{W}_{i}} \leq \nu_{2}\left(|x|_{\mathcal{W}}\right)
$$

for all $x \in M$. Since the set $\mathcal{W}$ is compact, then there are functions $\nu_{3}, \nu_{4} \in \mathcal{K}_{\infty}$ and a scalar $c_{0} \geq 0$ such that for all $x \in M$,

$$
|x| \leq \nu_{3}\left(|x|_{\mathcal{W}}\right)+c_{0},|x|_{\mathcal{W}} \leq \nu_{4}(|x|) .
$$

Thus, in this work we will consider the family (4) under Assumption 1 , i.e. a family of robustly stable nonlinear systems. In general, the sets $\mathcal{W}_{i}$ include equilibrium (at the origin, for instance) and limit cycles of agents in (4). There exist many works devoted to synchronization and design of consensus protocols for such a family or oscillatory network [26], [27], [28]. The goal of this study is to find a condition under which the existence of a global synchronization/consensus protocol for $d=0$ implies robust synchronization in (4) for a bounded $d \neq 0$.

Let a $\mathcal{C}^{1}$ function $y(x): M \rightarrow \mathbb{R}^{q}, y(0)=0$ be a synchronization measure for (4). We say that the family (4) is synchronized (or reached the consensus) if $y(x(t)) \equiv 0$ for all $t \geq 0$ on the solutions of the network under properly designed control actions

$$
u_{i}(t)=\varphi_{i}[y(x(t))]
$$

$\left(\varphi_{i}: \mathbb{R}^{q} \rightarrow \mathbb{R}^{m_{i}}\right.$ is a $\mathcal{C}^{1}$ function, $\left.\varphi_{i}(0)=0\right)$ for $d(t) \equiv 0$, $t \geq 0$. In this case the set $\mathcal{A}=\{x \in \mathcal{W}: y(x)=0\}$ contains the synchronous solutions of the unperturbed family in (4) and the problem of synchronization of "natural" trajectories is considered since $\mathcal{A} \subset \mathcal{W}$. In fact, due to the condition $\varphi_{i}(0)=0$ the convergence of $y$ (synchronization/consensus) implies that the solutions of the interconnection belong to $\mathcal{W}$, the conditions of convergence of the synchronizing/consensus output $y$ can be found in [26], [27], [28]. Therefore, it is assumed that the controls $\varphi_{i}(y)$ ensure the network global synchronization, while decomposability in general follows from Assumption 1. We are going to show that in the setup as above, by selecting the shapes of $\varphi_{i}$, it is possible to guarantee robust synchronization of (4) for any measurable and essentially bounded input $d$.

To this end, by continuity arguments there exist functions $\eta_{1}, \eta_{2}, \mu_{i} \in \mathcal{K}_{\infty}$ with a scalar $\eta_{0} \geq 0$ such that for all $x \in M$ :

$$
\begin{gathered}
|y(x)| \leq \eta_{0}+\eta_{1}\left(|x|_{\mathcal{W}}\right),|y(x)| \leq \eta_{2}(|x|), \\
\left|\varphi_{i}(y)\right| \leq \mu_{i}(|y|)
\end{gathered}
$$

(note that the first two inequalities are related through (7)). Then the following intermediate result can be established under Assumption 1 for (4), (8).

In this paper proofs are omitted due to space limitation.

Proposition 9. Let Assumption 1 be satisfied for (4). Then there exist $\varphi_{i}, i=1, \ldots, N$ in (8) such that the interconnection (4), (8) has $p G S$ property with respect to the set $\mathcal{W}$.

To proof the above proposition, we need the following restriction to satisfy:

$$
\mu_{i}(s) \leq \gamma_{u i}^{-1}\left[N^{-1} \alpha_{4} \circ \eta_{1}^{-1}(0.5 s)\right] .
$$

Assumption 2. For $\mu_{i}$ satisfying (10) and all $\varphi_{i}: \mathbb{R}_{+} \rightarrow \mathbb{R}_{+}$ that satisfy

$$
\left|\varphi_{i}(y)\right| \leq \mu_{i}(|y|), \forall y \in \mathbb{R}^{p},
$$

the compact set $\mathcal{A}$ contains all $\alpha$ and $\omega$ limit sets of (4), (8) for $d=0$, and it is decomposable.

Note that by definition of the set $\mathcal{A},|x(t)|_{\mathcal{W}} \leq|x(t)|_{\mathcal{A}} \leq$ $|x(t)|_{\mathcal{W}}+z$ for a scalar $z \geq 0$ for all $x \in M$, then the pGS property with respect to the set $\mathcal{A}$ has also been proven.

Therefore, in the setup used in this work the boundedness of trajectories (boundedness of $|x(t)|_{\mathcal{W}}$ implies the same property for $|x(t)|$ according to (7)) follows by a proper selection of the interconnection gain in (8), i.e. by decreasing the control gain a certain robustness of (4), (8) is inherited after individual systems as it is stated in Assumption 1.

Theorem 10. Let assumptions 1 and 2 be satisfied for (4), (8). Then there exist $\varphi_{i}, i=1, \ldots, N$ in (8) such that the interconnection (4), (8) has $A G$ property with respect to the set $\mathcal{A}$.

Roughly speaking this qualitative result says that if the synchronized output $y$ is related with $|x|_{\mathcal{W}}$ as in (9) and each system in the network is robustly stable as in Assumption 1, then the system can be robustly synchronized by a sufficiently small feedback proportional to $y$. 


\section{EXAMPLES AND SIMULATIONS}

\section{A. Application to nonlinear pendulums with friction}

This example is taken from [27]. Consider a network of nonlinear non-identical pendulums for $i=1, \ldots, N, N>1$ :

$$
\begin{aligned}
& \dot{x}_{1 i}=x_{2 i}, \\
& \dot{x}_{2 i}=-\Omega_{i}^{2} \sin \left(x_{1 i}\right)-\kappa x_{2 i}+d_{i},
\end{aligned}
$$

where the state $x_{i}=\left[x_{1 i}, x_{2 i}\right]$ takes values on the cylinder $M_{i}:=\mathbb{S} \times \mathbb{R}$, the exogeneous disturbance $d_{i}(t) \in \mathbb{R}, \kappa$ is a constant parameter and $\Omega_{i}^{2}$ is the angular frequency of individual pendulums. The unperturbed system has a Hamiltonian $H\left(x_{i}\right)=0.5 x_{2 i}^{2}+\Omega_{i}^{2}\left(1-\cos \left(x_{1 i}\right)\right)$ and $\dot{H}=x_{2 i} d_{i}-\kappa x_{2 i}^{2}$. Each unperturbed system has two equilibrium $[0,0]$ and $[\pi, 0]$ (the former is attractive and the later one is a saddle-point), thus $\mathcal{W}_{i}=\{[0,0] \cup[\pi, 0]\}$ is a compact set and containing all $\alpha$ - and $\omega$-limit sets of (11) for $d_{i}=0$. In addition, it is straightforward to check that $\mathcal{W}_{i}$ is decomposable in the sense of Definition 3.

Lemma 11. [29] For each $i=1, \ldots, N$, the systems in (11) is ISS with respect to the set $\mathcal{W}_{i}$.

Consequently, Assumption 1 is satisfied for (11) (since admitting an ISS Lyapunov function is equivalent to enjoying AG property according to Theorem 8) and we may select the synchronization measure $y$ for the network. Since, in [27], authors have considered the first coordinate as synchronization measure, we are going to follow the same idea. The synchronization measure in this work is:

$$
y=A \sin \left(x_{1}\right),
$$

where $x_{1}=\left[x_{11}, \ldots, x_{1 N}\right]^{T}$ and $A \in \mathbb{R}^{N \times N}$ is a Metzler matrix as in the first example.

Since the global boundedness of trajectories of (11) for bounded inputs is proven in Lemma 11, then a local analysis around equilibria is sufficient to show the synchronization measure convergence. It is straightforward to check that linearized around equilibria dynamics has $y=0$ as a stable and attractive manifold. By this, the convergence of $y$ is guaranteed locally. Then by taking,

$$
\varphi_{i}(y)=\beta y_{i}, \beta>0,
$$

we may suppose that Assumption 2 is satisfied for some sufficiently small $\beta$. The results of simulations confirm this conclusion which can be seen in Fig. 1 where a) is the disturbance free case and b) represents the simulation result with disturbances. Zoomed view of case-b can be seen in Fig. 2. The simulation parameters are $N=5, \Omega_{i}^{2}=0.02 i$, $\beta=0.1$ and the disturbance inputs are $\left[\phi_{1}, \ldots, \phi_{5}\right]^{T}=$ $[0.1 \sin (t),-0.15 \sin (t),-0.2 \sin (t), 0.15 \sin (t), 0.2 \sin (t)]^{T}$ and

$$
A=\left[\begin{array}{ccccc}
-3 & 1 & 1 & 0 & 1 \\
1 & -3 & 1 & 1 & 0 \\
1 & 1 & -3 & 1 & 0 \\
0 & 1 & 1 & -3 & 1 \\
1 & 0 & 0 & 1 & -2
\end{array}\right]
$$
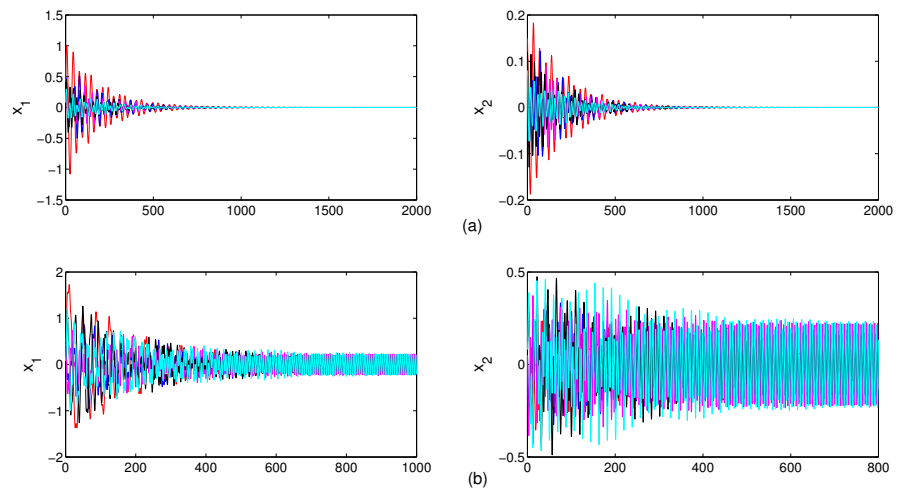

Figure 1. The result of simulation for (11)
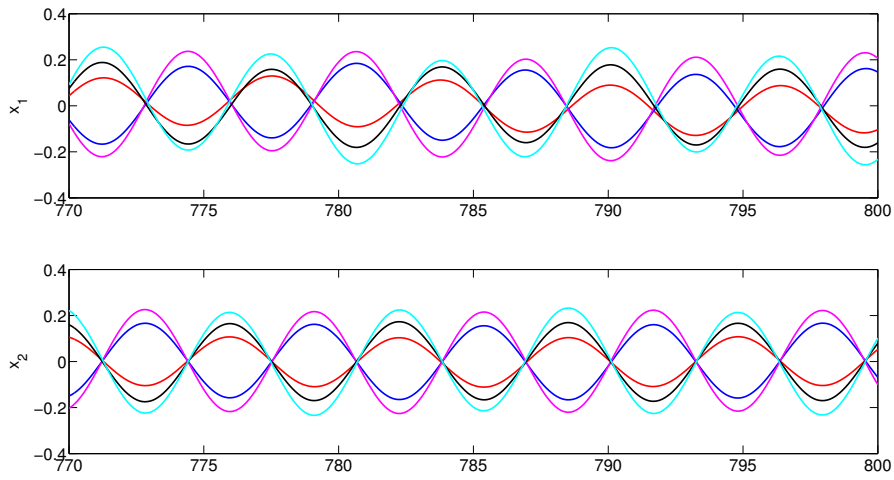

Figure 2. Zoomed view of case b) of Fig. 1.

\section{CONCLUSIONS}

Based on an extension of the ISS framework to systems with multiple invariant sets and evolving on (non-compact) a manifold, sufficient conditions for robust synchronization were derived. The condition imposed on the controller $\left(\varphi_{i}(0)=0\right)$ made sure that the convergence of the synchronization measure implies that the interconnection (system-controller) belongs to the decomposable set $\mathcal{W}$. Practical global stability analysis of the interconnection was done with respect to $\mathcal{W}$. The asymptotic gain property of the interconnection with respect to the set of synchronous solutions $\mathcal{A}(\mathcal{A} \subset \mathcal{W})$ was also proved.

Numerical simulations proved the effectiveness of our method to network of both identical and nonidentical nodes. However, our results are applicable only to systems that allow decomposition without cycles.

\section{REFERENCES}

[1] N. Kuznetsov, G. Leonov, H. Nijmeijer, and A. Pogromsky, "Synchronization of two metronomes," in Proceedings of the 3rd IFAC Workshop on Periodic Control Systems, vol. 3, 2007.

[2] I. I. Blekhman, Synchronization in science and technology. ASME Press, 1988.

[3] G. V. Osipov, J. Kurths, and C. Zhou, Synchronization in Oscillatory Networks. Springer, 2007.

[4] A. Pikovsky and J. Kurths, Synchronization: A Universal Concept in Nonlinear Sciences. Cambridge University Press, 2003.

[5] S. H. Strogatz, Sync: How Order Emerges from Chaos in the Universe, Nature, and Daily Life. Hyperion, 2004.

[6] F. Dorfler and F. Bullo, "Synchronization and transient stability in power networks and nonuniform kuramoto oscillators," SIAM Journal on Control and Optimization, vol. 50, no. 3, pp. 1616-1642, 2012. 
[7] R. Wei and R. Beard, Distributed Consensus in Multi-vehicle Cooperative Control. Communications and Control Engineering, Springer, 2008.

[8] V. Gazi and K. M. Passino, Swarm Stability and Optimization. Springer, 2011.

[9] J. M. González-Miranda, Synchronization and Control of Chaos: An Introduction for Scientists and Engineers. Imperial College Press, 2004.

[10] F. Lewis, H. Zhang, K. Hengster-Movric, and A. Das, Cooperative Control of Multi-Agent Systems. Communications and Control Engineering, Springer, 2014.

[11] J. S. Shamma, Cooperative Control of Distributed Multi-Agent Systems. Wiley-Interscience, 2008.

[12] R. Olfati-Saber, "Flocking for multi-agent dynamic systems: algorithms and theory," Automatic Control, IEEE Transactions on, vol. 51, no. 3, pp. 401-420, 2006.

[13] S. Dashkovskiy, D. Efimov, and E. Sontag, "Input to state stability and allied system properties," Automation and Remote Control, vol. 72, no. 8, pp. 1579-1614, 2011.

[14] D. Angeli, J. Ferrell, and E. Sontag, "Detection of multistability, bifurcations and hysteresis in a large class of biological positive-feedback systems," Proc. Natl. Acad. Sci. USA, vol. 101, pp. 1822-1827, 2004.

[15] D. Angeli and E. Sontag, "Multi-stability in monotone input/output systems," Systems \& Control Lett., vol. 51, pp. 185-202, 2004.

[16] A. Gelig, G. Leonov, and V. Yakubovich, Stability of nonlinear systems with non unique equilibrium. Moscow: Nauka, 1978. [in Russian].

[17] G.-B. Stan and R. Sepulchre, "Analysis of interconnected oscillators by dissipativity theory," IEEE Trans. Automatic Control, vol. 52, pp. 256270, 2007.

[18] G. A. Leonov and N. V. Kuznetsov, "Hidden attractors in dynamical systems. from hidden oscillations in hilbert-kolmogorov, aizerman, and kalman problems to hidden chaotic attractor in chua circuits," International Journal of Bifurcation and Chaos, vol. 23, no. 01, 2013.

[19] M. Chaves, T. Eissing, and F. Allgower, "Bistable biological systems: A characterization through local compact input-to-state stability," IEEE Trans. Automatic Control, vol. 45, pp. 87-100, 2008.

[20] A. Rantzer, "A dual to Lyapunov's stability theorem," Syst. Control Lett., vol. 42, pp. 161-168, 2001.

[21] D. Angeli, "An almost global notion of input-to-state stability," IEEE Trans. Automatic Control, vol. 49, pp. 866-874, 2004.

[22] D. Angeli and D. Efimov, "On input-to-state stability with respect to decomposable invariant sets," in Proc. 52nd IEEE Conference on Decision and Control, (Florence), 2013.

[23] D. Efimov, "Global lyapunov analysis of multistable nonlinear systems," SIAM Journal on Control and Optimization, vol. 50, no. 5, pp. 31323154, 2012.

[24] Z. Nitecki and M. Shub, "Filtrations, decompositions, and explosions," American Journal of Mathematics, vol. 97, no. 4, pp. 1029-1047, 1975.

[25] J. Guckenheimer and P. Holmes, "Structurally stable heteroclinic cycles," Math. Proc. Camb. Phil. Soc., vol. 103, pp. 189-192, 1988

[26] Z. Li, Z. Duan, G. Chen, and L. Huang, "Consensus of multiagent systems and synchronization of complex networks: A unified viewpoint," Circuits and Systems I: Regular Papers, IEEE Transactions on, vol. 57, no. 1, pp. 213-224, 2010.

[27] J. Zhao, D. Hill, and T. Liu, "Global bounded synchronization of general dynamical networks with nonidentical nodes," Automatic Control, IEEE Transactions on, vol. 57, no. 10, pp. 2656-2662, 2012.

[28] A. Y. Pogromsky, "A partial synchronization theorem," Chaos, vol. 18, p. 037107, 2008.

[29] D. Efimov, R. Ortega, and J. Schiffer, "Iss of multistable systems with delays: application to droop-controlled inverter-based microgrids," in Proc. ACC 2015, Chicago, 2015. 